\title{
ON HOLONOMY AND HOMOGENEOUS SPACES
}

\author{
BERTRAM KOSTANT
}

1.1. Introduction. In general a homogeneous space admits many invariant affine connections. Among these are certain connections which appear in many ways to be more natural than the others. We refer to the connections which K. Nomizu in [4] calls canonical affine connections of the first kind. When $G$ is a compact connected Lie group and $K$ a closed subgroup we called an invariant Riemannian metric on $G / K$, natural (in [2]) when it induced such a connection.

The papers [2] and [3] were devoted mainly to the determination of the holonomy group and its reducibility properties for $G / K$ when the latter is provided with a natural invariant metric. The theorems obtained generalize completely (to such homogeneous spaces $G / K$ ) the results of E. Cartan on holonomy in Riemannian symmetric homogeneous spaces.

The purpose of this paper is mainly to consider questions of holonomy when $G / K$ is provided with an arbitrary invariant metric and not just a natural one. In this connection we are concerned with how reducibility properties change when we change from one metric to another. In $\$ 2$ and $\$ 3$ here we determine the linear holonomy group as well as the linear holonomy algebra for an arbitrary invariant metric. This is done in terms of the adjoint representation and a comparison operator between the arbitrary metric and a natural one. We then show the restricted holonomy group and the full homogeneous holonomy both admit the same invariant subspaces with regard to their respective actions on a tangent space of $G / K$.

In $\$ 4$ we order the various metrics with regard to their reducibility properties. We then obtain conditions on an arbitrary metric which permits comparison between its reducibility properties and the reducibility properties of a natural metric. (The point being that the situation in the latter case is

Received November 27, 1956.

Revised March 6, 1957. 
known completely as a result of Theorem 5, [3].) These comparison theorems are applied in various ways throughout the remainder of the paper.

1.2. One of the applications of the main comparison theorem is to the case, considered by Lichnerowicz [6], where the isotropy group $K$ has inequivalent tangential representations. In [5] Lichnerowicz asserts a result on reducibility for $G / K$ which, roughly speaking, is similar though actually weaker than our Theorem 5, [3]. However, Lichnerowicz makes no assumption as to the type of invariant metric whereas we had taken it to be natural. In a later paper, however, [6], he writes that his theorem is based on a result of Nomizu; a result, he says, which now appears doubtful. He then obtains a similar theorem to his original one only after making the assumption on the isotropy group referred to above. In this paper we will, first of all, show that the original theorem of Lichnerowicz is indeed false. (We do this, in $\S 3$, by exhibiting a homogeneous space $G / K$ which is reducible even though $G$ is simple. This then is a counter-example to the conjecture of Nomizu. As we have shown in [3], Corollary 9, such a situation cannot occur if the metric is natural.) Next as an application of the comparison theorem we show that the assumption on the isotropy group implies that all metrics on $G / K$ have the exact same reducibility property. Thus Theorem 5, [3], may be applied and this yields a stronger result than his corrected result.

1.3. In [7] Matsushima and Hano prove that $G / K$ is always irreducible whenever $G$ is simple and the Euler characteristic of $G / K, \chi(G / K)$, is not zero. After being informed of this fact by Matsushima we recognized that if $\chi(G / K)$ $\neq 0$ then $K$ satisfies the condition of $\S 1.2$. In fact, as we show in $\S 5, K$ actually satisfies a stronger condition. We are then able to apply the comparison theorem and obtain the result of [7] (referred to above) and more, namely that when $\chi(G / K) \neq 0, G / K$ is irreducible if and only if $G$ is simple. (Of course the new "only if" part is valid only when the natural assumption is made that the Lie algebra of $G$ acts effectively on $G / K$.)

As an application we obtain the corollary that a simple and a non-simple Lie group cannot both act transitively and effectively as a group of motions on a compact Riemannian manifold $M$ if $\%(M) \neq 0$. This result is not true without the assumption, $\%(M) \neq 0$.

1.4. In $\S 6$ a comparison theorem is applied to show that under mildly. 
restrictive circumstances the "least" reducibility for $G / K$ is achieved with a natural metric. An example is given to show, however, that a natural metric does not have this property in the general case.

2.1. The holonomy algebra. Let $G$ be a compact connected Lie group. Let $g$ be its Lie algebra, identified with the tangent space at the identity $e \in G$. We assume the bracket relation is defined using the right invariant vector fields on $G$.

Let $K \cong G$ be a closed subgroup and let $¥$ be its Lie algebra. Let $p \subseteq g$ be a subspace such that $\mathfrak{g}=\mathfrak{f}+\mathfrak{p}$ is a direct sum and such that $\mathfrak{p}$ is invariant under $a d u$ for all $u \in K$. For any $X \in \mathfrak{g}$ let $X_{\mathfrak{f}}$ and $X_{\mathfrak{p}}$ be such that $X=X_{\mathfrak{f}}+X_{\mathfrak{p}}$ where $X_{\mathfrak{p}} \in$ and $X_{\mathfrak{p}} \in \mathfrak{p}$. Also for any $X \in \mathfrak{g}$ let $D_{X}$ be the operator on $p$ defined by $D_{X}(Y)=[X, Y]_{p}$ for all $Y \in \mathfrak{p}$.

Let $G / K$ be the space of left cosets of $K$. Let $o \in G / K$ be the point corresponding to the coset $K$. As usual we identify $p$ with the tangent space at $o$. For any $X \in g$ let $\tilde{X}$ be the vector field on $G / K$ whose value at $q \in G / K$ is defined by

$$
\left.\widetilde{X} f\right|_{q}=\lim _{t \rightarrow 0} \frac{f(\exp t X \cdot q)-f(q)}{t}
$$

where $f$ is any smooth function defined in a neighborhood of $q$ and the dot designates the usual action of $G$ on $G / K$.

It is immediate that $[\widetilde{X, Y}]=[\widetilde{X}, \widetilde{Y}]$.

2.2. Let a homogeneous Riemannian metric be given on $G / K$. The vector fields $\widetilde{X}, X \in g$, then become infinitesimal motions on $G_{/} / K$.

For any $X \in \mathfrak{g}$ let $a_{X}: \mathfrak{p} \rightarrow \mathfrak{p}$ be the operator on $\mathfrak{p}$ defined by $a_{X} Y=-\Gamma_{1}(\widetilde{X})$ for all $Y \in \mathfrak{p}$. Here $\nabla_{Y}$ designates covariant differentiation at $o$ by the vector $Y \in \mathfrak{p}$.

Now let $s_{o}$ be the linear holonomy algebra of $G / K$ at $o$. By Corollary 4.5, [1], $\tilde{\Xi}_{0}$ is exactly the Lie algebra generated by all $a_{\lambda}$ for $X \in \mathfrak{a}$. Hence we wish to compute $a_{X}$ for all $X \in g$. Let $X, Y \in \mathfrak{g}$, then

$$
[X, Y]_{\mathfrak{p}}=\nabla_{x_{\mathfrak{p}}} \tilde{Y}-\nabla_{x_{\mathfrak{p}}} \tilde{X} \text {. }
$$

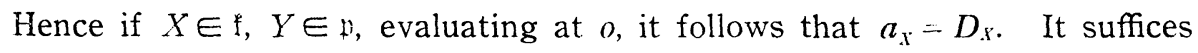
then to determine $a_{X}$ when $X \in \mathfrak{p}$. 
If $u, v$ are tangent vectors at a point $q \in G / K$ let $\{u, v\}$ designate their inner product as given by the metric tensor.

Lemma 2.2. Let $X, Y, Z \in \mathfrak{p}$. Then

$$
2\left\{a_{z} X, Y\right\}=\left\{D_{z} X, Y\right\}-\left\{D_{Z} Y, X\right\}-\left\{[X, Y]_{\mathfrak{p}}, Z\right\}
$$

Proof. Using the metric we may identify vector fields with Pfaffian forms.

We recall the well known evaluation of the differential of a Pfaffian form $\tilde{Z}$ on a pair of vectors

$$
d \widetilde{Z}(Y, X)=Y\{\tilde{X}, \widetilde{Z}\}-X\{\tilde{Y}, \widetilde{Z}\}-\left\{[Y, X]_{\mathfrak{p}}, Z\right\} .
$$

Evaluating the first two terms using covariant differentiation we see that

$$
d \tilde{Z}(Y, X)=\left\{X, \nabla_{Y} \widetilde{Z}\right\}-\left\{Y, \nabla_{X} \widetilde{Z}\right\}=2\left\{a_{Z} X, Y\right\} .
$$

On the other hand evaluating the first two terms of (1) using Lie differentiation we have

$$
d \tilde{Z}(Y, X)=\left\{X,[Y, Z]_{\mathfrak{p}}\right\}-\left\{Y,[X, Z]_{\mathfrak{p}}\right\}+\left\{[Y, X]_{\mathfrak{p}}, Z\right\} .
$$

Comparing (a) and (b) proves the lemma.

Q.E.D.

2.3. Let $C$ designate the bilinear form $\{X, Y\}$ at $o$. It is clear that $D_{X}$ is skew-symmetric with respect to $C$ if $X \in *$. This need not be the case if $X \in \mathfrak{p}$. If this were the case it follows immediately from Lemma 2.2 that $a_{Z}=\frac{1}{2} D_{z}$ for $Z \in \mathfrak{p}$.

On the other hand if $B^{*}$ is a bilinear form on $g$, invariant under $a d G$, and $B$ is its restriction to $\mathfrak{p}$ then assuming $B$ is positive definite on $\mathfrak{p}$ and that $\mathfrak{p}$ is orthogonal to with respect to $B^{*}$ it is obvious that $D_{X}$ is skew-symmetric for all $X \in \mathfrak{g}$.

Forgetting about $B^{*}$ we will say that a positive definite bilinear form $B$ on $\mathfrak{p}$ is strictly invariant if $D_{X}$ is skew-symmetric for all $X \in g$. It is not necessarily true that all complements $\mathfrak{p}$ admit such a $B$ but we may always choose $\mathfrak{p}$ such that a strictly invariant $B$ exists on $\mathfrak{p}$.

Assume $B$ is a strictly invariant bilinear form on $\mathfrak{p}$. We designate by $(X, Y)$ its value on $X, Y \in \mathfrak{p}$. Returning to our metric, let $S$ be the operator on $\mathfrak{p}$ (positive definite with respect to $B$ ) defined by

$$
(S X, Y)=\{X, Y\}
$$


for all $X, Y \in \mathfrak{p}$.

Now by Lemma 2.2

$$
2\left(S a_{Z} X, Y\right)=\left(S D_{Z} X, Y\right)-\left(D_{Z} Y, S X\right)-\left(D_{X} Y, S Z\right) .
$$

Since $D_{Z}$ and $D_{X}$ are skew-symmetric relative to $B$ and $D_{X}(S Z)=-D_{S Z} X$ we have

$$
2\left(S a_{Z} X, Y\right)=\left(S D_{Z} X, Y\right)+\left(D_{Z} S X, Y\right)-\left(D_{S Z} X, Y\right)
$$

so that

$$
2 S a_{Z}=S D_{Z}+D_{Z} S-D_{S Z}
$$

Hence $a_{Z}=\frac{1}{2}\left(D_{z}+S^{-1} D_{Z} S-S^{-1} D_{s Z}\right)$ for any $Z \in \mathfrak{p}$.

But now $S$ commutes with $D_{Z}$ for any $Z \in{ }^{*}$. Hence setting $S=0$ on ${ }^{*}$ and recalling that $a_{Z}=D_{Z}$ for all $Z \in \mathfrak{f}$ we see that

$$
a_{Z}=\frac{1}{2}\left(D_{Z}+S^{-1} D_{Z} S-S^{-1} D_{s Z}\right)
$$

for all $Z \in g^{1)}$ Hence recalling Corollary 4.5 in [1] we have

TheOREM 2.3. Let $G / K$ be given any homogeneous Riemannian metric. Let $\mathfrak{p}$ be any complement to $\mathfrak{f}$ such that $\mathfrak{p}$ admits a strictly invariant bilinear form $(X, Y)$. Let $\{X, Y\}$ be the bilinear form on $p$ given by the metric tensor. Let $S: p \rightarrow p$ be defined by $(S X, Y)=\{X, Y\}$. Extend $S$ to a by defining $S=0$ on 4 . Now for any $Z \in \mathfrak{g}$ let $D_{Z}$ be the operator on $p$ defined by $D_{Z} Y=[Z, Y]_{\mathrm{p}}$ for all $Y \in \mathfrak{p}$.

Then the linear holonomy algebra is the Lie algebra generated by all operators on $p$ of the form

$$
D_{Z}+S^{-1} D_{Z} S-S^{-1} D_{S Z}
$$

where $Z \in$ g. $^{11}$

3.1. The counter-example and the holonomy group. Now, of course, if we fix $\mathfrak{p}$ and $B$ and vary $C$ through all possible bilinear forms in $p$ invariant under $a d u, u \in K$, we run through all possible homogeneous metrics that we may put on $G / K$. In considering the question as to how $a_{L}$ changes from metric to

1) Of course $S$ becomes singular in general when extended from $p$ to $g$. We understand $S^{-1}$ to be the operator on $p$ which is inverse to the restriction of $S$ to $p$. 
metric or how the holonomy algebra changes from metric to metric we are reduced to seeing how

$$
D_{z}+S^{-1} D_{z} S-S^{-1} D_{s z}
$$

changes as we vary $S$ through all possible operators which are positive definite on $\mathfrak{p}$ with respect to $B$ and which commute with $a d u$ for all $u \in K$.

3.2. Let us then consider the question of reducibility of $G / K$ and how this depends on the choice of the permissible metric $C .^{2)}$ For example, we might ask the question, if $G / K$ is irreducible relative to one permissible metric when can we be sure it will remain irreducible when the metric is changed.

Now in [2] and [3] all our considerations were for the case when $C=B$. Concerning reducibility among the theorems proved was one, Corollary 9, [3], asserting that if $g$ is simple then $G / K$ is necessarily irreducible.

Now one may wonder whether $G / K$ is always irreducible if $g$ is simple (that is, always, independent of the invariant metric). In fact such a theorem unknown to us at the time [3] was written had at one time been claimed by Nomizu. An even more general result had been announced in a paper of Lichnerowicz (Theorem, parts $1^{\circ}, 3^{\circ}$, p. 1413, [5]). ${ }^{3)}$ We now construct a counterexample showing that these theorems are false. That is, we shall give an example of a case where $g$ or $G$ is simple yet $G / K$ is reducible:

We first observe the following fact. Let $G$ and $K$ be as before. Let $H$ be any compact connected subgroup of $G$. Let $\mathfrak{h}$ be its Lie algebra. Consider the action of $H$ on $G / K$. We assert, first of all, that a necessary and sufficient condition that $H$ be transitive on $G / K$ is that $g=\mathfrak{h}+\mathfrak{f}$. Indeed this is almost obvious. If $g=\mathfrak{h}+\mathfrak{f}$ then $H$ carries $o \in G / K$ into a set which is both open and closed in $G / K$. By connectivity then the orbit of $o$ must be $G / K$. Conversely, if $H$ is transitive an obvious argument on dimension implies that $\mathfrak{g}=\mathfrak{h}+\mathfrak{l}$.

Now many examples exist where a simple compact Lie algebra $g$ is the sum (not necessarily direct) of two proper subalgebras $\mathfrak{h}$ and $\mathfrak{f}$. For example, the 6 -sphere $S^{6}$ may be written $S^{6}=S O(7) / S O(6)$. But $G_{2}$ (the first exceptional compact Lie group) is contained in $S O(7)$ and acts transitively on $S^{6}$ so that if $a$ is the Lie algebra of $S O(7)$ and $\mathfrak{f}$ and $\mathfrak{h}$ the Lie algebras of $G_{2}$ and $S O(6)$

2) Any positive definite bilinear form $C$ on $p$ which is invariant under $a d_{p} K$ will be called a permissible metric from here on.

3) See, [6] for a new, but weaker, version of this theorem. 
we have such an example. Another example is where Spin(9) acts transitively on the 15-dimensional sphere.

Assume then that $\mathfrak{B}=\mathfrak{h}+\mathfrak{f}$ where of course it may be assumed that both $H$ and $K$ are compact. Let $V=G / H \times G / K$. Obviously we may put a metric on $V$ which is reducible and which is invariant under $G \times G$. Let $\hat{G}$ be the diagonal of $G \times \mathrm{G}$. It is obvious that $\hat{G}$ is isomorphic to $G$ and that $\hat{G}$ leaves the metric on $V$ invariant. We assert that $\hat{G}$ acts transitively on $V$ so that $V=\hat{G} / L$. Indeed writing $V=G \times G / H^{o} \times K^{o}$ where $H^{o}=H \times e$ and $K^{o}=e \times K$. Then where $\mathfrak{h}^{o}, \mathfrak{f}^{o}$ and $\hat{g}$ are the respective Lie subalgebras of $g \oplus g$ it is clear that $\mathfrak{f}^{o}+\mathfrak{h}^{o}+\hat{g}=g \oplus g$.

Q.E.D.

3.3. Returning to our considerations of reducibility in the general case let $s_{0}(C)$ be the holonomy algebra, $\psi_{o}(C)$ the linear holonomy group and $\tau_{o}(C)$ the restricted linear holonomy group at $o$ induced by the metric $C$. Of course $\tau_{o}(C)=\exp \unlhd_{o}(C)$.

Now in [2], $\psi_{o}(B)$ has been determined as well as $\tau_{o}(B)$ (that is, without assumptions such as simple connectivity for $G$ or connectedness for $K$ ). To be more explicit we recall, first of all, that for any permissible metric $C, \tau_{o}(C)$ is the identity component of $\psi_{o}(C)$. Obviously $a d_{p} K^{*} \leqq \tau_{o}(C)$ where $K^{*}$ is the identity component of $K$ and $a d_{\mathfrak{p}} K^{*}$ is the restriction of $a d K^{*}$ to $\mathfrak{p}$. We have shown in [3] that when $C=B, \psi_{o}(B)=a d_{\mathfrak{p}} K \cdot \tau_{o}(B)$. That is, first of all, $a d_{\mathfrak{p}} K$ $\leqq \psi_{o}(B)$ and secondly every coset of $\tau_{o}(B)$ in $\psi_{o}(B)$ contains an element of $a d_{\mathfrak{p}} K$. Hence if

$$
K^{\prime}=\left\{u \in K \mid a d_{\mathfrak{p}} u \in \tau_{o}(B)\right\}
$$

then $K^{\prime}$ is a normal subgroup of $K$ containing $K^{*}$, and $K / K^{\prime}$ is naturally isomorphic with $\psi_{o}(B) / \tau_{o}(B)$. We now wish to show all of this is true for an arbitrary metric $C$.

Let $\delta \subseteq g$ be the subset defined by

$$
\delta=\{X \in g \mid \exp X \in K\} .
$$

If $\pi: G \rightarrow G / K$ is the natural map let $X(t), 0 \leqq t \leqq 1$, be the closed curve in $G / K$, where $X(0)=X(1)=o$, defined by $X(t)=\pi(\exp t X)$. Let $a(X) \in \psi_{0}(C)$ be the element in $\psi_{o}(C)$ defined by parallel transport along $X(t)$. For simplicity write $u(X)=\exp X \in K$. We have shown in [2] that 


$$
a(X)=a d_{\mathfrak{p}} u(-X) \cdot \exp a_{X} \cdot{ }^{4)}
$$

Thus $a d_{\mathfrak{p}} u(X)=\exp a_{X} \cdot a(X)^{-1}$. Since $a_{X} \in \xi_{0}(C)$ it follows then that $a d_{p} u(X) \in \psi_{o}(C)$ for all $X \in \delta$. Since any $u \in K$ is of the form $\exp X$ for $X \in \mathfrak{g}$ it follows that $a d_{p} K \leqq \psi_{o}(C)$. On the other hand we assert that every element of the fundamental group $\Pi_{1}(G / K, o)$ has a representative, some curve $X(t)$ where $X \in \delta$. In fact we may choose $X \in \mathfrak{p}$. This is true since if we use the metric $B$ instead of $C$ the curves $\pi(\exp s Y)$ where $Y \in \mathfrak{v}$ and $(Y, Y)=1$ are all geodesics through $o$ and every geodesic through $o$ (parameterized by the arc length) is necessarily of this form. Now as pointed out in [2] every element of $\Pi_{1}(G / K, o)$ has as representative, a closed geodesic through $o$. This is an easy consequence of the homogeneity of $G / K$ and the classical theorem that every free homotopy class contains a closed geodesic. But if $\pi(\exp s Y)$ is closed where we may assume $0 \leqq s \leqq b$ then of course $b Y=X \in \emptyset \cap p$ and $X(t)=\pi(\exp t b Y), 0 \leqq t \leqq 1$.

Hence if $a \in \psi_{o}(C)$ is arbitrary we may find $X \in \delta \cap p$ such that the curve defining $a \in \psi_{0}(C)$ and the curve $X(t)$ are homotopic. Hence $a(X)^{-1} a \in \tau_{o}(C)$. Hence $a \in a(X) \cdot \tau_{o}(C)$. But by (3.3.1) we have $a \in a d_{\mathfrak{p}} K \cdot \tau_{o}(C)$. We have proved

THEOREM 3.3. Let $C$ be an arbitrary permissible metric. Let $\Xi_{0}(C)$ be the corresponding holonomy algebra (given explicitly by Theorem 2.3) so that $\exp B_{i}(C)=\tau_{0}(C)$ is the restricted linear holonomy group. Let $\psi_{0}(C)$ be the full linear holonomy group. Then $a d_{\mathfrak{p}} K \leqq \psi_{o}(C)$ and in fact

$$
\psi_{o}(C)=a d_{\mathfrak{p}}(K) \cdot \tau_{o}(C) .
$$

3.4. We wish to show now that the concept of reducibility is independent of whether we consider $\psi_{o}(C)$ or $\tau_{o}(C)$. More explicitly, we will show that a subspace $\mathfrak{p}^{\prime} \subseteq \mathfrak{b}$ is invariant under $\tau_{0}(C)$ if and only if it is invariant under $\psi_{o}(C)$. Furthermore, we will show that an element of $\mathfrak{p}^{\prime}$ is fixed under the action of $\tau(C)$ if and only if it is fixed under the action of $\psi_{0}(C)$.

We record for later use the following immediate consequence of the relation (2.1.1).

4) Actually, in [2] we have been dealing with the case where $C=B$. However the proof of this formula makes no use of this fact. Thus the formula holds for an arbitrary invariant metric $C$. 
By the definition of $a_{z}$ for any $Z \in \mathfrak{g}$ we have

$$
[X, Y]_{\mathfrak{p}}=a_{X} Y_{\mathfrak{p}}-a_{Y} X_{\mathfrak{p}}
$$

for any $X, Y \in g$.

Some lemmas are required.

LemmA 3.4A. Let $\mathfrak{p}_{1} \leqq \mathfrak{p}$ be any subspace invariant under the action of $\tau_{o}(C)$. Let $\mathfrak{p}_{2}$ be its $(C)$ orthogonal complement in $\mathfrak{p}$. Let $\mathfrak{h}_{1}=\mathfrak{f}+\mathfrak{p}_{1}$ and $\mathfrak{h}_{2}=\mathfrak{l}+\mathfrak{p}_{2}$. Then $\mathfrak{h}_{1}$ and $\mathfrak{h}_{2}$ are subalgebras of $\mathfrak{g}$.

Proof. Of course $[\mathfrak{k}, \mathfrak{k}] \subseteq \mathfrak{l}$ and $\left[\mathfrak{f}, p_{1}\right] \subseteq p_{1}$. Now let $X, Y \in p_{1}$. Then by (3.4.1), $[X, Y]_{\mathfrak{p}}=a_{X} Y-a_{Y} X . \quad$ But by Theorem 3.3, [1], $a_{X}, a_{Y} \in \Xi_{0}(C)$ and hence $a_{X} Y, a_{Y} X \in \mathfrak{p}_{1}$. Thus $[X, Y]_{\mathfrak{p}} \in \mathfrak{p}_{1}$ so that $[X, Y] \in \mathfrak{l}_{1}$. Thus $\mathfrak{l}_{1}$ is a subalgebra. Similarly, since $\mathfrak{p}_{2}$ is invariant under $\tau_{o}(C), \mathfrak{h}_{2}$ is a subalgebra.

Q.E.D.

Let $\mathfrak{p}_{1}, \mathfrak{p}_{2}, \mathfrak{h}_{1}$ and $\mathfrak{h}_{2}$ be as above. Consider the direct sum $\mathfrak{g}=\mathfrak{p}_{1}+\mathfrak{h}_{2}$. Let $Q: \mathfrak{g} \rightarrow p_{1}$ be the projection of $g$ onto $p_{1}$ which vanishes on $\mathfrak{f}_{2}$.

Now one cannot expect that $D_{X}$ is skew-symmetric on $p$ for all $X \in q$ (indeed by Lemma 4.2 , it follows easily that this is the case if and only is $B$ and $C$ induce the same affine connection). This situation, however, is somewhat approached (see proof of Lemma 3.4B below) by the fact that if $X \in \mathfrak{l}$ ): then $Q \cdot a d X$, as an operator on $w_{1}$, is skew-symmetric. This forms the main idea behind the following lemma. Now let $H_{1}$ (resp. $H_{2}$ ) be the ciosure in $G$ of the subgroup corresponding to the subalgebra $\mathfrak{h}_{1}\left(\right.$ resp. $\left.\mathfrak{h}_{2}\right)$. We will adhere to this notation throughout this section.

Lemma 3. 4 B. Let $Y \in \mathcal{g}$ and $x \in H_{2}$. Then

$$
(Q Y, Q Y)=(Q(a d x Y), Q(a d x Y)) .
$$

Moreover, if $x$ is of the form $x=\exp ^{-} X$ for $X \in \mathfrak{h}_{2}$, then $Q(\operatorname{adx} Y)=\exp a_{X}(Q Y)$.

Proof. Let $X \in \mathfrak{h}_{2}$ and $Z \in \mathfrak{p}_{1}$. Then $[X, Z]_{\mathfrak{p}}=a_{X} Z-a_{Z} X_{\mathfrak{p}}$. But since $a_{x}, a_{Z} \in \Xi_{o}(C)$ by Theorem 3.3, and since $X_{\mathfrak{p}} \in \mathfrak{p}_{2}$ we have that $a_{X} Z \in p_{1}$ and $a_{Z} X_{\mathfrak{p}} \in p_{:}$. Thus we may write $[X, Z]=a_{X} Z+\left([X, Z]_{\mathfrak{f}}-a_{Z} X_{\mathfrak{p}}\right)$ where the first term is in $\mathfrak{p}_{1}$ and the term within the parentheses is contained in $\mathfrak{h}_{2}$. Thus $\operatorname{QadX} Z=a_{x} Z$ and hence $Q \operatorname{adX}=a_{x}$ on $\mathfrak{h}_{1}$. On the other hand since $\mathfrak{h}_{\text {. }}$ is a subalgebra we have in fact that 


$$
Q(a d X)^{n}=\left(a_{X}\right)^{n} Q
$$

on $g$ for any integer $n$ and hence $Q a d x=\exp a_{X} Q$. (It should be recalled that since $a d X$ is defined by means of right invariant vector fields on $G$, $\exp a d X$ $=a d \exp -X$.) But since $a_{X}$ is skew-symmetric on $p_{1}$ (and hence $\exp a_{X}$ is orthogonal on $p_{1}$ ) it follows that

$$
(Q Y, Q Y)=(Q(a d x Y), Q(\operatorname{adx} Y))
$$

when $x$ is of the form $\exp X$ for some $X \in \mathfrak{h}_{2}$. Since we may substitute $\operatorname{adx} Y$ for $Y$ and choose other elements $x$ in $H_{2}$ of this form it follows easily that (3.4.2) is true for all $x$ in the group corresponding to $\mathfrak{h}_{2}$. By taking limits it is true whenever $x \in H_{2}$.

Now it is easy to see that every element $y \in G$ is of the from $y=u_{2} u_{1}$ where $u_{1} \in H_{1}, u_{2} \in H_{2}$. Indeed since $g=\mathfrak{h}_{1}+\mathfrak{h}_{2}$ it follows, see $\S 3.2$, that $H_{2}$ is transitive on $G / H_{1}$. This, however, is equivalent to the statement that every $y \in G$ is of the form, $y=u_{2} u_{1}$ where $u_{2} \in H_{2}, u_{1} \in H_{1}$.

Lemma 3.4C. Assume $y \in K$ and $y=u_{2} u_{1}$ where $u_{1} \in H_{1}$ and $u_{2} \in H_{2}$;

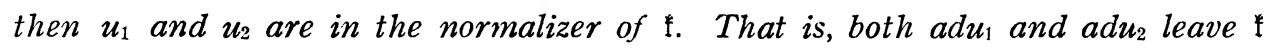
invariant.

Proof. Obviously it suffices to prove the lemma for just $u_{1}$. Let $Z \in \mathbb{f}$ and let $a d u_{1} Z=Y=Y_{1}+Y_{2}$ where $Y_{1} \in \mathfrak{f}, Y_{2} \in \mathfrak{p}$. Since it is obvious that $a d u_{1}$ leaves $\mathfrak{h}_{1}$ invariant we must have $Y_{2} \in \mathfrak{p}_{1}$. But now by Lemma 3.4B where $x=u_{2}$

$$
\begin{aligned}
\left(Q\left(a d u_{2} Y\right), Q\left(a d u_{2} Y\right)\right) & =(Q Y, Q Y) \\
& =\left(Y_{2}, Y_{2}\right) .
\end{aligned}
$$

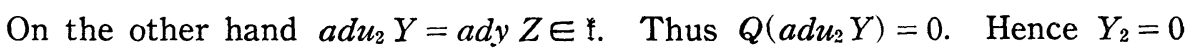
so that $Y \in \mathfrak{*}$. Thus $\mathfrak{l}^{\mathfrak{a}}$ is invariant under $a d u_{1}$.

Q.E.D.

Finally,

Lemma 3.4D. Let $V$ be a finite dimensional real vector space. Let $C_{1}$ and $C_{2}$ be two positive definite bilinear forms on $V$ given, respectively, by $(u, v)_{1}$ and $(u, v)_{2}$ for $u, v \in V$. Let $A$ be an operator on $V$ which is orthogonal with respect to $C_{1}$ and such that

$$
(u, u)_{2} \gtrsim(A u, A u)_{2}
$$

for all $u \in V$. Then $A$ is also orthogonal with respect to $C_{2}$. 
Proof. Let $T$ be the operator on $V$, positive definite with respect to $C_{1}$, defined by $(T u, v)_{1}=(u, v)_{2}$ for all $u, v \in V$. Assume $u \in V$ where $(w, w)_{2}$ $>(A w, A w)_{2}$. Since $w \neq 0$ we may assume $(w, w)_{1}=1$. Let $e_{i}, i=1,2, \ldots, n$ be a basis of $V$ which is (1) orthonormal with respect to $C_{1}$ and (2) such that $e_{1}=w$. Now

$$
\begin{aligned}
\operatorname{tr} A^{-1} T A & =\sum_{i=1}^{n}\left(A^{-1} T A e_{i}, e_{i}\right)_{1} \\
& =\sum_{i=1}^{n}\left(T A e_{i}, A e_{i}\right)_{1} \\
& =\sum_{i=1}^{n}\left(A e_{i}, A e_{i}\right)_{2}
\end{aligned}
$$

and

$$
\begin{aligned}
\operatorname{tr} T & =\sum_{i=1}^{n}\left(T e_{i}, e_{i}\right)_{1} \\
& =\sum_{i=1}^{n}\left(e_{i}, e_{i}\right)_{2}
\end{aligned}
$$

But $\left(e_{i}, e_{i}\right)_{2} \geqslant\left(A e_{i}, A e_{i}\right)_{2}$ and $\left(e_{1}, e_{1}\right)_{2}>\left(A e_{1}, A e_{1}\right)_{2}$. Thus $\operatorname{tr} T>\operatorname{tr} A^{-1} T A$ which is a contradiction. Thus $(w, w)_{2}=(A w, A w)_{2}$ for all $w \in V$. Q.E.D.

We now have

THEOREM 3.4. A subspace $\mathfrak{p}_{1} \subseteq \mathfrak{p}$ is invariant under $\tau_{0}(C)$ if and only if it is invariant under $\psi_{o}(C)$. Furthermore the elements of $\mathfrak{p}_{1}$ are fixed under the action of $\tau_{o}(C)$ if and only if they are fixed under the action of $\psi_{o}(C)$.

Proof. Assume $p_{1}$ is invariant under $\tau_{0}(C)$. To show $p_{1}$ is invariant under $\psi_{o}(C)$ it suffices by Theorem 3.3 to show that $p_{1}$ is invariant under $a d_{p} K$.

Now it is obvious that if Theorem 3.4 is proved in terms of a complement $p$ the theorem is true for any complement $\mathfrak{p}^{\prime}$ to which is invariant under $a d K$. It is convenient for this proof to choose $p$ as the orthogonal complement to $f$ relative to a positive definite invariant bilinear form $B^{*}$ on $g$.

Now let $y \in K$, then as mentioned above $y$ may be written as $y=u_{2} u_{1}$ where $u_{1} \in H_{1}$ and $u_{2} \in H_{2}$. But by Lemma 3.4 $\mathrm{C} a d u_{2}$ and $a d u_{1}$ leave invariant. But then by the definition of $\mathfrak{p}$ they also leave $\mathfrak{p}$ invariant. Now let $Z \in \mathfrak{p}_{1}$; we wish of course to show that $a d y Z \in \mathfrak{p}_{1}$. Now let $W=a d u_{1} Z$. Then since both $\mathfrak{p}$ and $\mathfrak{h}_{1}$ are invariant under $a d u_{1}$ we have that $W \in \mathfrak{h}_{1} \cap \mathfrak{p}=\mathfrak{p}_{1}$. Thus $\mathfrak{p}_{1}$ is invariant under $a d u_{1}$. Let $A$ be the restriction of $a d u_{1}$ to $\mathfrak{p}_{1}$. Let $Y=a d y Z$ $=a d u_{2} W$. Write $Y=Y_{1}+Y_{2}$ where $Y_{1} \in \mathfrak{p}_{1}$ and $Y_{2} \in \mathfrak{p}_{2}$. Now by Lemma 3.4B, 
$(W, W)=\left(Y_{1}, Y_{1}\right)$. On the other hand $y \in K$ and hence $a d v$ is orthogonal with respect to $C$. Thus

$$
\begin{aligned}
(Z, Z) & =(Y, Y) \\
& =\left(Y_{1}, Y_{1}\right)+\left(Y_{2}, Y_{2}\right) \\
& =(W, W)+\left(Y_{2}, Y_{2}\right) .
\end{aligned}
$$

But then since $W=A Z$ we have by (I) that

$$
(Z, Z) \gtrsim(A Z, A Z)
$$

for all $Z \in \mathfrak{p}_{1}$. On the other hand $A$ is orthogonal with respect to the restriction of $B^{*}$ to the subspace $p_{1}$. Thus by Lemma 3.4D $A$ is orthogonal with respect to $C$. Hence $(Z, Z)=(W, W)$ and hence by (I), $Y_{2}=0$. Thus $Y \in w_{1}$ and hence $\mathfrak{p}_{1}$ is invariant under $a d K$.

Now assume that every vector in $p_{1}$ is invariant under the action of $\tau_{o}(C)$. Then for any $Y \in \mathfrak{g}$ the range of $a_{Y}$ is contained in $\mathfrak{p}_{2}$. Thus if $X, Z \in \mathfrak{g}$ then $[X, Z]_{\mathfrak{p}}=a_{X} Z_{\mathfrak{p}}-a_{Z} X_{\mathfrak{p}} \in \mathfrak{p}_{2}$ and hence $[X, Y] \in \mathfrak{h}_{2}$. Thus $\mathfrak{h}_{2}$ is an ideal in $\mathfrak{g}$ which contains the commutator ideal. Thus if $Z \in p_{1}$ it follows that $Q(\operatorname{adX})^{n} Z$ $=0$ for all $n>0$ and of course equals $Z$ when $n=0$. Thus since every $x$ in $G$ is of the form $\exp X$ for some $X \in \mathfrak{g}$ we have $Q(a d x Z)=Z$ for all $x \in G$. In particular if $x \in K$ then since $a d x Z \in$ p we may write $a d x Z=Z+W$ where $W \in p_{2} . \quad$ On the other hand since $(\operatorname{adx} Z, a d x Z)=(Z, Z)$ it follows that $W=0$ and hence $a d x Z=Z$ for all $x \in K$ so that $Z$ is invariant under the action of $\psi_{o}(C)$ by Theorem 3.3.

Q.E.D.

4.1. The reducibility equivalence theorem. Now we wish to compare the reducibility properties of the various permissible metrics on $\mathfrak{p}$. For this purpose it is convenient to partially order the metrics. We will say $C_{2}<C_{1}$ if whenever a subspace $\mathfrak{p}_{1} \subseteq \mathfrak{p}$ is invariant under $\psi_{0}\left(C_{1}\right)$ it is also invariant under $\psi_{0}\left(C_{2}\right)$. In particular we wish to compare $C$ with $B$ when $C$ is arbitrary. This is true mainly because we completely know the reducibility properties of $B$.

Now let $\mathfrak{p}=\sum_{i=0}^{m} \mathfrak{p}_{i}(C)$ be the direct sum where $p_{0}(C)$ is the set of all vectors in $p$ which are fixed by $\psi_{o}(C)$ and $p_{i}(C), i=1,2, \ldots, m(C)$ are irreducibly invariant subspaces under the action of $\psi_{o}(C)$. By Theorem 3.4 we would get the exact same decomposition of $\mathfrak{p}$ had we used $\tau_{o}(C)$ instead of $\psi_{o}(C)$. Since the representations of $\tau_{o}(C)$ on the subspaces $\dot{p}_{i}(C)$ are known to be inequivalent it follows obviously that the representations of $\psi_{0}(C)$ on these subspaces are 
also inequivalent. Thus a subspace $p^{\prime} \equiv \downarrow$ is invariant under $\psi_{0}(C)$ if and only if $\mathfrak{p}^{\prime}$ is of the form $\mathfrak{p}^{\prime}=\mathfrak{p}^{\prime} \cap p_{0}(C)+\sum_{j-1}^{k} p_{i j}(C)$ where $i_{j}, j=1,2, \ldots, k$ runs through some subset of the numbers $i=1,2, \ldots, m(C)$. One sees then that $C_{1}<C_{2}$ if and only if $\mathfrak{h}_{i}\left(C_{2}\right)$ is invariant under $\psi_{0}\left(C_{1}\right)$ for all $i$ and $\mathfrak{b}_{0}\left(C_{2}\right)$ $\leqq \mathfrak{p}_{0}\left(C_{1}\right)$. We will say $C_{1}$ is weakly equivalent to $C_{2}$ or $C_{1} \sim C_{2}$ whenever $C_{1}<C_{2}$ and $C_{2}<C_{1}$. It is immediately clear that $C_{1} \sim C_{2}$ if and only if $p_{0}\left(C_{1}\right)$ $=\mathfrak{p}_{0}\left(C_{2}\right)$ and for any $i \geqslant 1$ there exists $j \geqslant 1$ such that $\mathfrak{p}_{i}\left(C_{1}\right)=\mathfrak{p}_{j}\left(C_{2}\right)$. That

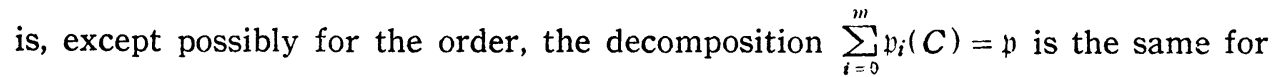
both $C_{1}$ and $C_{2}$.

4.2. It is convenient to introduce, finally, a strong equivalence for the permissible metrics. We will say $C_{3} \cong C_{2}$, or $C_{1}$ is strongly equivalent to $C_{2}$ if they induce the same affine connection on $G / K$. Obviously, if $C_{1} \cong C_{2}$ then $\psi_{o}\left(C_{1}\right)=\psi_{o}\left(C_{2}\right)$, since the holonomy group depends solely on the connection. Hence, of course, $C_{1} \cong C_{2}$ implies $C_{1} \sim C_{2}$. A useful criterion for strong equivalence is given in

Lemma 4.2. Let $C_{1}$ be any permissible metric and let $\sum_{i=0}^{m} \mathfrak{v}_{i}\left(C_{1}\right)=p$ be the associated decomposition of $\mathfrak{p}$. Let $C_{2}$ be any positive definite bilinear form on $\mathfrak{p}$ and let $T: \mathfrak{p} \rightarrow \mathfrak{p}$ be the operator defined by $\{T X, Y\}_{1}=\{X, Y\}_{2}$.

Then $C_{2}$ is a permissible metric which is strongly equivalent to $C_{1}$ if and only if $T$ commutes with $\dot{\varphi}_{0}\left(C_{1}\right)$. (That is, if and only if, (1) $T$ leaves $w_{i}\left(C_{1}\right)$ invariant for all $i$ and (2) if $T_{i}$ is the restriction of $T$ to $b_{i}\left(C_{1}\right)$ then eash $T_{i}$ reduces to a scalar $\lambda_{i}$ when $i>0$ ).

Proof. If $C_{2}$ is a permissible metric strongly eqnivalent to $C_{1}$ then $\dot{\psi}_{o}\left(C_{1}\right)$ leaves $C_{1}$ and $C_{2}$ invariant. Hence $\psi_{0}\left(C_{1}\right)$ commutes with $T$.

Conversely, if $T$ commutes with $\psi_{0}\left(C_{1}\right)$ then by Theorem $3.3, C_{2}$ is invariant under $a d_{p} K$ so that $C_{2}$ is a permissible metric. Furthermore, if $\widetilde{C}_{2}$ is the invariant metric on $G / K$ defined by $C_{2}$ and $\widetilde{T}$ is defined on $G / K$ accordingly then $\nabla_{X} \widetilde{T}=-\left[a_{x}, T\right]$ for any $X \in g$ (See $\$ 2.2$ ). Here $\nabla_{x}$ and $a_{x}$ are defined relative to $C_{1}$. This follows from the fact that $L_{x}=\Gamma_{x}+a_{x}$ (See [1], \$2.1) where $L_{x}$ designates Lie differentiation relative to the field $\widetilde{X}$ where the value is computed at $o$. But by the invariance of $\widetilde{T}, L_{X} \widetilde{T}=0$.

But now since $a_{x} \in B_{0}\left(C_{1}\right)$ by Theorem 3.3 in [1] and $\psi_{0}\left(C_{\mathrm{i}}\right)$ commutes with $T$ it follows that $\nabla_{、} \widetilde{T}=0$. By invariance then it follows that $\widetilde{T}$ is covariant 
constant. Thus $\widetilde{C}_{2}$ is covariant constant relative to the affine connection induced by $C_{1}$. By the uniqueness of the affine connection induced by $C_{2}$ in that it is torsion-free and $\widetilde{C}_{2}$ is covariant constant it follows that $C_{1}$ and $C_{2}$ induce the same affine connection.

Q.E.D.

4.3. Now consider the question as to when $C<B$ where $C$ is an arbitrary metric. It is suggestive from the results of [3] and also from the example of $\S 3.2$ that $C<B$ in most cases. Actually as illustrated in the example of $\S 5.1$ this relation is not true in general. However, it is always true under mildly restrictive circumstances. For example, see $\S 6.1$, it is always true when $K$ is connected, $\mathfrak{p}$ is chosen properly, and there are no non-zero invariant vector fields.

Now since $a_{X}=\frac{1}{2} D_{X}$ when $B=\mathrm{C}$ it is clear from Theorems 3.3 and 2.3 and the remarks in $\S 4.1$ that $C<B$ whenever $S$ leaves the subspaces $\mathfrak{p}_{i}(B)$, $i=0,1,2, \ldots, m(B)$ invariant. To prove the converse we first observe

Lemma 4.3. For any permissible metric $C$ one always has

$$
\operatorname{dim} \mathfrak{p}_{0}(C) \leqq \operatorname{dim} \mathfrak{p}_{0}(B) .
$$

Proof. Where $C$ is arbitrary let $q(C)=\sum_{i=1}^{m} p_{i}(C)$ so that $p=p_{0}(C)+q(C)$ is a direct sum. Obviously it suffices to show that $q(B) \cong q(C)$. But by Theorems 2.3 and 3.4 it follows that $q(B)$ is the linear space spanned by all vectors in $p$ of the form $D_{X} Y=[X, Y]_{\mathfrak{p}}$ where $X \in \mathfrak{g}, Y \in \mathfrak{p}$. On the other hand $[X, Y]_{\mathfrak{p}}=a_{X} Y-a_{Y} X_{\mathfrak{p}} \in \mathfrak{q}(C)$ so that $q(B) \subseteq q(C)$.

Now if $C<B$ then first of all $\mathfrak{p}_{0}(B) \subseteq \mathfrak{p}_{0}(C)$. But by Lemma 4.3 this implies $\mathfrak{p}_{0}(B)=p_{0}(C)$. But then we see that each subspace $\mathfrak{p}_{i}(B), i>0$ may be written as a direct sum of certain of the subspaces $\mathfrak{p}_{j}(C)$ where $j>0$. Since the subspaces $\mathfrak{p}_{j}(C)$ are mutually orthogonal with respect to $C$ it follows that the subspaces $\mathfrak{p}_{i}(B)$ are also mutually orthogonal with respect to $C$. This, however is equivalent to the condition that $S$ leaves the subspace $p_{i}(B)$ invariant. We have proved

Theorem 4.3. Let $C$ be any permissible metric. Then $C<B$ if. and only if $S$ leaves the subspaces $\mathfrak{p}_{i}(B)$ invariant, $i=0,1, \ldots, m(B)$.

4.4. We now consider the question as to when $B<C$. Of course the counter-example given in $\$ 3.2$ for the reducibility of $G / K$ when $G$ is simple is 
a situation where $B<C$ does not hold. One would like to know conditions on $C$ so as to insure $B<C$. Such conditions are given in Theorem 4.4. First, however, we need

Lemma 4.4A. Let $\mathfrak{p}_{1} \leqq \mathfrak{p}$ be a subspace invariant under $\psi_{0}(C)$. Then if $S$ leaves $p_{1}$ invariant, $p_{1}$ is also invariant under $\psi_{0}(B)$. Moreover if the elements of $\mathfrak{p}_{1}$ are invariant under $\psi_{o}(C)$ they are also invariant under $\psi_{0}(B)$.

Proof. Let $\mathfrak{b}_{2}, \mathfrak{h}_{1}$ and $\mathfrak{h}_{2}$ be as in Lemma 3.4A. According to that lemma $\mathfrak{h}_{1}$ and $\mathfrak{h}_{2}$ are subalgebras of $\mathfrak{g}$. Thus $D_{X}$ leaves $\mathfrak{p}_{1}$ invariant for every $X \in \mathfrak{p}_{1}$. Now if $S$ leaves $p_{1}$ invariant then $p_{1}$ and $p_{2}$ are orthogonal relative to $B$. Thus $D_{X}$ leaves $p_{2}$ invariant also. That is, for any $Z \in p_{2}, D_{x} Z \in p_{2}$. But $D_{x} Z$ $=-D_{Z} X$ and for similar reasons $D_{Z}$ leaves $p_{1}$ invariant, hence $[X, Z]_{p} \in$ $\mathfrak{p}_{1} \cap \mathfrak{p}_{2}=0$. Thus $D_{Z} X=0$. But then $\mathfrak{p}_{1}$ is invariant under $D_{Z}$ for all $Z \in \mathfrak{g}$. Since of course $p_{1}$ is invariant under $a d_{p} K$ it follows that $p_{1}$ is invariant under $\psi_{0}(B)$.

Now if the elements of $\mathfrak{p}_{1}$ are invariant under $\psi_{0}(C)$ then where $X \in \mathfrak{h}_{1}$ and $Y \in \mathfrak{p}_{1},[X, Y]_{\mathfrak{p}}=a_{X} Y-a_{Y} X_{\mathfrak{p}}=0$ since $X_{\mathfrak{p}} \in \mathfrak{p}_{1}$ and $a_{X}, a_{Y} \in \mathfrak{g}_{0}(C)$. Thus $D_{X}$ vanishes on $\mathfrak{p}_{1}$ when $X \in \mathfrak{h}_{1}$. We already know that $D_{X}$ vanishes on $\mathfrak{p}_{1}$ when $X \in p_{2}$. Thus since $p_{1}$ is element-wise invariant under $a d_{p} K$ it is also elementwise invariant under $\psi_{o}(B)$.

Q.E.D.

It follows as an immediate consequence of Lemma 4.4 that $B<C$ whenever $S$ leaves the subspaces $\mathfrak{p}_{j}(C)$ invariant for all $j$. Unlike the converse in the case when we were dealing with $C<B$ the converse in this case is not necessarily true. This is so because of the arbitrary nature of $S$ on $p_{0}(B)$. The condition $B<C$ implies that $\mathfrak{p}_{0}(C) \leqq \mathfrak{p}_{0}(B)$. However, as the example of $\S 6.1$ illustrates $p_{0}(B)$ may actually be larger than $p_{0}(C)$ and since $p_{0}(B)=\sum_{j} p_{0}^{j}(B)$ where $p_{0}^{j}(B)=p_{0}(B) \cap p_{j}(C)$ it is perfectly possible that the subspaces $p_{0}^{i}(B)$ are not mutually orthogonal with respect to $B$. On the other hand we may alter $B$ (and hence $S$ ) on $\mathfrak{p}_{0}(B)$, preserving its strict invariance, and obtain $B^{\prime}$ (and $S^{\prime}$ ) which is strongly equivalent to $B$ (see Lemma 4.2) and which is such that $S^{\prime}$ leaves the subspaces $p_{j}(C)$ invariant.

We now observe

Lemma 4.4B. Let $\mathrm{c}$ be the center of $\mathrm{g}$. Then $\mathfrak{p}_{0}(B)=\mathfrak{c} \cap \mathfrak{p}$.

Proof. It is obvious that $c \cap p \subseteq p_{0}(B)$. On the other hand if $X \in p_{0}(B)$ and $Y \in \mathbb{Q}$ then $D_{\mathfrak{Y}} X=[Y, X]_{\mathfrak{p}}=0$ so that $[X, Y] \in \mathfrak{f}$. On the other hand 
$\left[f, v_{0}(B)\right]=0$. Thus $(a d X)^{2} Y=0$ for all $Y \in \mathfrak{g}$. But $a d X$ is a completely reducible operator on 9. Hence $(a d X)^{2}=0$ implies $a d X=0$ or $X \in$ c. $\quad$ Q.E.D.

Collecting the results in this section we have proved

Theorem 4.4. Let $C$ be any permissible metric. Then $B<C$ whenever $S$ leaves the subspaces $\mathfrak{p}_{j}(C)$ invariant. Conversely, if $B<C$ we may find a permissible strictly invariant metric $B^{\prime}$ on $\mathfrak{p}$ which is strongly equivalent to $B$ and which is such that $S^{\prime}$ (see above) leaves the spaces $\mathfrak{n}_{j}(C)$ invariant.

If we assume $\mathfrak{p} \cap \mathrm{c}=0$, where $\mathrm{c}$ is the center of $\mathrm{g}$, then $B<C$ if and onlv if $S$ leaves the subspaces $p_{j}(C)$ invariant.

Corollary 4.4. Assume a is semi-simple. Let $C$ be any permissible metris on $\mathfrak{p}$. Then $B<C$ if and only if $S$ leaves the subspaces $\mathfrak{w}_{j}(C)$ invariant.

4.5. Combining Theorems 4.3 and 4.4 we see that $B \sim C$ whenever $S$ leaves the spaces $p_{j}(C)$ and $p_{i}(B)$ invariant. On the other hand if $B \sim C$ then since we may assume (see $\S 4.1) p_{i}(B)=p_{i}(C)$ for all $i$, the subspaces $p_{i}(B)$ are mutually orthogonal with respect to both $C$ and $B$. Hence $S$ leaves these spaces invariant. This gives

Corollary 4.5. Let $C$ be any permissible metric. Then $B \sim C$ if and only if $S$ leaves all the subspaces $\mathfrak{n}_{i}(B)$ and $\mathfrak{p}_{j}(C)$ invariant.

4.6. Now in order to apply Cor. 4.5 one would like to have conditions which assert that either (1) $S$ leaves each $p_{j}(C)$ invariant or $(2) S$ leaves each $H_{i}(B)$ invariant: for example, $(1)$ is true if $S$ is in the associative algebra generated by $\psi_{0}(C)$. Similarly $(2)$ is true if $S$ is in the associative algebra generated by $\psi_{0}(B)$. A situation in which both (1) and (2) occur is as follows: Lichnerowicz uses the expression (which we adopt), " $a d_{p} K$ has inequivalent representations" when every irreducible representation of $a d_{p} K$ has, at most, multiplicity 1 on $\mathrm{k}$. Now it is an elementary fact that if $a d_{\mathfrak{p}} K$ (or any compact linear group) has inequivalent representations then whenever a subspace $\mathfrak{p}_{1} \subseteq \mathfrak{p}$ is invariant under $a d_{\mathfrak{p}} K$ any operator on which commutes with $a d_{\mathfrak{p}} K$ must also leave $\mathfrak{p}_{1}$ invariant. Since $S$ always commutes with $a d_{\mathfrak{p}} K$ it follows that if $a d_{\mathfrak{n}} K$ has inequivalent representations then $S$ leaves both $\mathfrak{p}_{j}(C)$ and $\mathfrak{p}_{i}(B)$ invariant for all $i, j$. Thus by Corollary $4.5 \mathrm{~B} \sim \mathrm{C}$ for all $C$. We thus cbtain

THEOREM 4.6. Assume ad $K$ has inequivalent representations. Then any 
tu'o permissible metrics $C_{1}$ ans $C_{\text {. }}$ are weakl equivalent. That is, the decomposition $y=\sum_{i=n}^{m} \mathfrak{b}_{i}(C)$ is independent of the metric $C$.

In [6] Lichnerowicz has shown that if $a d_{1} K$ has inequivalent representations and $G / K$ is simply connected then $G$ contains a connected normal subgroup which is the direct product of the subgroup $I^{x}$ which leave invariant the various "feuille" $W_{a}$. Also the subgroups $i^{\cdot x}$ act effectively and transitively on the $W_{\alpha}$."

Thus by making assumptions on the representation of $a d n$, Lichnerowicz obtains a result for an arbitrary metric on $G / K$ which is roughly similar (though actually weaker) than our Theorem $5,[3]$, where no assumptions have been made on the representation of $a d_{F} K$ but our metric is taken to be of a special type $(C=B)$. But now we see as a result of Theorem 4.6 the assumption made by Lichnerowicz implies that all metrics are equivalent as to their reducibility properties. Thus with this assumption our Theorem $j,[3]$, can be applied to give a stronger result than that in [6]. We have in Lie algebra terms,

COROLlary 4.6. Let $B$ be an invariani (underad $G$ ) positive definite bilinear form on $\mathrm{a}$. Let b be the ortho-compienent of t. Nou assume a acts effertively on $G / K$ and assume $a d_{p} K$ has inequivalent retresentations.

Let $C$ be nuy permissible metric on $\mathrm{b}$ and let fol (C) be the holonomy group associated with the innariant Riemannian meris on G/K induced by $C$.

Then (1) $v_{1} \leqq n$ is invariant under $\psi_{0}(C)$ if and only if $g\left(p_{1}\right)=b_{1}+\left[p_{1}, b_{1}\right]$ is an ideal in \&. In such a case $\$\left(b_{1}\right)=f_{1}+b_{1}$ where $f_{1}=g\left(b_{1}\right) \cap \mathrm{f}$.

Furthermore if $\mathrm{p}=\sum_{2=0}^{\prime \prime} \mathrm{b}_{i}(C)$ is the direci sum decomposition of $\mathrm{b}$ assoriated with $C$ (see 5.1$)$ then where $n_{2}=H_{i}(C)$

(2) $\left.n_{2}=n_{i}+\Gamma n_{i}, n_{i}\right], i=0,1,2, \ldots, m$ are idcals in $\mathrm{a}$ and $\mathrm{a}=\square_{0} \oplus, n_{1} \oplus \ldots$ $\oplus g_{m}$ is direct. Also,

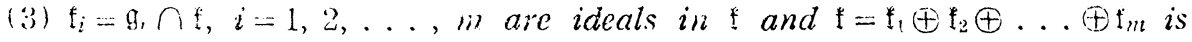
direct. Finally,

(4) $g_{0}=b_{0}=n \cap c$ where $c$ is the center of $\mathrm{g}$.

5.1. Applications. It is an immediate consequence of Corollary 4.6 that

5 Although not stated explicitly as it was in [5] it seems clear that the author has assumed $G$ acts effectively on $G, K$. One can easily construct counter-examples when $G$ does not act effectively. 
$G / K$ is irreducible when the following two conditions are satisfied: (1) $g$ is simple and (2) $a d_{p} K$ has inequivalent representations.

An important example of when (2) is always satisfied is when the Euler characteristic of $G / K(\%(G / K))$ is not zero (see Theorem 5.3 below). Thus one obtains the result that if $g$ is simple and $\%(G / K) \neq 0$ then $G / K$ is always irreducible. The applicability of Corollary 4.6 to obtain this result occurred to us only after being informed by Matsushima that this result had been proved (using other methods) in [7]. However, as we shall see below, Corollary 4.6 yields more than this; namely, if we assume that $g$ acts effectively in $G / K$ (i.e.,

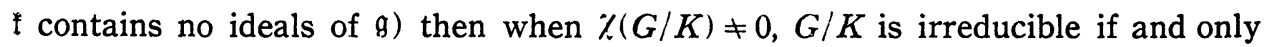
if $g$ is simple. ${ }^{6)}$ More generally, the subspaces $p_{j}(C)$ are in a natural one-one relation with the simple ideals in $g$.

5. 2. Let $a d_{\mathfrak{i}}$ and $a d_{\mathfrak{p}}$ designate the respective representations of $K$ on $\mathfrak{f}$ and $\mathfrak{p}$ obtained by restricting $a d K$ to these spaces. We will say that $a d_{\mathfrak{f}}$ and $a d_{\mathfrak{p}}$ are disjoint if every irreducible component of $a d_{\mathfrak{l}}$ is not equivalent to any irreducible component of $a d_{\mathfrak{k}}$.

We recall that an intertwining operator $T$ for a pair of representations $\sigma_{1}$ and $\sigma_{2}$ of $K$ on the respective vector spaces $V_{1}$ and $V_{2}$ is a linear operator $T: V_{1} \rightarrow V_{2}$ such that $T \sigma_{1}(x)=\sigma_{2}(x) T$ for all $X \in K$. In the above sense $\sigma_{1}$ and $\sigma_{2}$ are disjoint if and only if the only intertwining operator is the one mapping $V_{1}$ into zero.

For the sake of convenience we shall assume in this section that $g$ acts effectively on $G / K$. (This is not as restrictive as the assumption that $G$ acts effectively on $G / K$.)

Lemma 5.2. Assume $a d_{\mathfrak{p}}$ and $a d_{\mathfrak{1}}$ are disjoint. Then

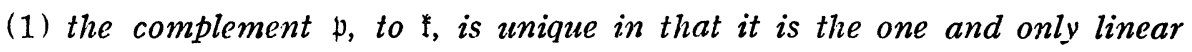
complement which is invariant under ad $K$,

(2) if $\mathfrak{g}^{\prime}$ is any ideal in $\mathfrak{g}$ then $\mathfrak{g}^{\prime}=\mathfrak{g}^{\prime} \cap \mathfrak{f}+\mathfrak{g}^{\prime} \cap \mathfrak{p}$,

(3) if $g_{0}$ is the center of $g$ and $\mathfrak{B}=\sum_{i=0}^{s} g_{i}$ is the direct sum where the $g_{i}, i>0$, are the simple ideals in $\mathrm{g}$ then $\mathfrak{p}=\sum_{j=0}^{s} \mathfrak{p}_{j}$ is a direct sum where $\mathfrak{p}_{j}=\mathfrak{p} \cap g_{j}$ and the subspaces $\mathfrak{p}_{j}$ are identical with the subspaces $\mathfrak{p}_{i}(B)$ in some order.

6) This means that if $X \in \mathfrak{g}, \widetilde{X}=0$ implies $X=0$. 
Proof. Let $a \leqq g$ be any invariant subspace for $a d K$. Let $a d_{\mathfrak{a}}$ be the representation of $K$ obtained by restricting $a d K$ to a. Now assume $a d_{\mathfrak{a}}$ is irreducible. Then $a d_{\mathfrak{a}}$ is equivalent to one of the irreducible components of either $a d_{\mathfrak{1}}$ or $a d_{\mathfrak{p}}$ but not both. Without loss of generality assume $a d_{\mathfrak{a}}$ is not equivalent to any irrebucible component of $a d_{\mathfrak{p}}$. Now for any $X \in \mathfrak{a}$ consider the linear mapping $X \rightarrow X_{\mathfrak{p}}$ of $a$ into $\mathfrak{p}$. This defines an intertwining operator $T$ for the representations $a d_{\mathfrak{a}}$ and $a d_{\mathfrak{p}}$. But since these representations are disjoint we must have $X_{\mathfrak{p}}=0$ for all $X \in \mathfrak{a}$ so that $\mathfrak{a} \leqq \ddagger$. Similarly if $a d_{\mathfrak{a}}$ and $a d_{\mathfrak{f}}$ are disjoint it follows that $a \subseteq \mathfrak{p}$. Thus we see that if $a d_{\mathfrak{a}}$ is irreducible then either $\mathfrak{a} \subseteq \mathfrak{f}$ or $\mathfrak{a} \leqq \mathfrak{p}$. More generally then, if $a d_{\mathfrak{a}}$ is not necessarily irreducible, by decomposing it into irreducible summands, we see that $a d_{\mathfrak{a}}$ may be uniquely written as the direct sum $\mathfrak{a}=\mathfrak{a}_{1}+\mathfrak{a}_{2}$ where $\mathfrak{a}_{1}=\mathfrak{a} \cap \mathfrak{f}$ and $\mathfrak{a}_{2}=\mathfrak{a} \cap \mathfrak{p}$.

Now if $p^{\prime}$ is a linear complement to ${ }^{\prime}$ which is invariant under adK then it is clear that $a d_{\mathfrak{p}}$, is equivalent to $a d_{\mathfrak{p}}$ and hence $\mathfrak{b}=\mathfrak{b}^{\prime}$. Also from above, $g^{\prime}=g^{\prime} \cap f+g^{\prime} \cap p$. It should be noted that if $g^{\prime} \neq 0$ then $g^{\prime} \cap p \neq 0$ since otherwise $g^{\prime} \cong \mathfrak{f}$ which contradicts our assumption that $g$ acts effectively on $G / K$.

It follows immediately then that $p=-\sum_{i=0}^{\infty} p_{i}$ is direct sum and that $n_{i} \neq 0$ for $i>0$. Furthermore the $\mathfrak{p}_{j}$ are invariant under $\psi_{0}(B)$. On the other hand if $p^{\prime}$ is invariant under $\psi_{o}(B)$ then obviously $a^{\prime}=\left[p^{\prime}, p^{\prime}\right]+b^{\prime}=f^{\prime}+h^{\prime}$ where $f^{\prime}=g^{\prime} \cap f$ is an ideal in $g$. Hence the $p_{i}$ are irreducibly invariant under $\psi_{0}(B)$. But since $p_{0}=p_{0}(B)$ by Lemma $4.4 B$ the subspaces $p_{j}$ must be identical with the subspaces $\mathfrak{p}_{i}(B)$ in some order.

Q.E.D.

5. 3. Now both the condition that $a d_{p} K$ has inequivalent representations and the condition $a d_{\mathfrak{f}}$ and $a d_{\mathfrak{p}}$ are disjoint are conditions on $K$ as a subgroup of $G$. We will say that a compact subgroup $K$ is detached when both these conditions are satisfied. In other words $K$ is a detached subgroup of $G$ when the irreducible components of $a d_{\mathfrak{p}}$ occur not only with multiplicity one in $a d_{\mathfrak{p}}$ but with multiplicity one in $a d_{g}$. One sees easily that if $K$ is a detached subgroup of $G$ then any compact subgroup $K^{\prime}$ of $G$ which contains $K$ is also detached.

We now observe

Lemma 5.3. Let $T$ be a maximal torus in $G$. Then $T$ is a detached sub. group. 
Proof. Let $¥$ be the Lie algebra of $T$. Then as one knows we may find a complement $\mathfrak{u}$ such that $\mathfrak{u}=\sum_{i=1}^{r} \mathfrak{y}_{i}$ is direct sum where $r$ is half the number of roots of the complexification of $\mathfrak{g}, \mathfrak{b}_{i}$ is two-dimensional for each $i$ and $\mathfrak{b}_{i}$ is irreducibly invariant under the action of $a d T$. Furthermore the subspaces $\mathfrak{v}_{i}$ are in one-one correspondence with the set of all pairs consisting of a root and its negative. This correspondence is such that the representation of $T$ on the complexification of $\mathfrak{b}_{i}$ is a direct sum of the character representations of $T$ defined by the root and its negative. Since two different roots define inequivalent character representations of $T$ it follows that the representations of $T$ on the various planes $\mathfrak{v}_{i}$ are inequivalent. Furthermore since $a d_{\mathfrak{t}}$ is just a multiple of the identity representation, $T$ is therefore detached.

Q.E.D.

Now one knows that $\%(G / K) \neq 0$ if and only if $K$ contains a maximal torus of $G$. Hence by Lemma 5.3 and the remark preceeding it $K$ is detached whenever $\chi(G / K) \neq 0$. Combining the results of Corollary 4.6 and Lemma 5.2 or more directly Theorem 4.6 and Lemma 5.2 we obtain the following theorem.

THEOREM 5.3. Assume $\%(G / K) \neq 0^{-}$and that a acts effectively on $G / K^{6)}$ Then with respect to any invariant metric, $G / K$ is irreducible if and only if $\mathrm{g}$ is simple.

More generally, $\mathrm{g}$ is necessarily semi-simple and if $\mathrm{g}=\sum_{i=1}^{s} g_{i}$ is the decomposition of $\mathrm{g}$ as the direct sum of its simple ideals then $\mathfrak{p}=\sum_{j=1}^{s} \mathfrak{p}_{j}$ is direct where $\mathfrak{p}_{j}=\mathfrak{p} \cap \mathfrak{g}_{j}$ and the subspaces $\mathfrak{p}_{j}$ are identical with the subspaces $\mathfrak{p}_{i}(C)$ in some order.

One should note that we may substitute the condition $K$ is detached for the condition $\%(G / K) \neq 0$ in the previous theorem.

5. 4. Of course it is quite possible for a compact Riemannian manifold $M$ to admit as a transitive, effective group of motions both a simple Lie group and a non-simple Lie group. An example of this is when $M$ is itself a compact non-abelian simple Lie group $G$ which is provided with a left and right invariant metric. Then $G$ and $G \times G$ modulo a finite group both act transitively and effectively as motions on $G$. The following corollary says this cannot happen if $\%(M) \neq 0$. The proof follows immediately from Theorem 5.3.

Corollary 5.4. A simple and a non-simple compact Lie group cannot both 
act transitively and effectively as a group of motions on a compact Riemannian manifold $M$ if $\%(M) \neq 0$.

5.5. For homogeneous spaces $G / K$ where $\%(G / K) \neq 0$ the relation between invariant metrics and affine connections they induce becomes quite direct. We have

Corollary 5.5. Assume $\%(G / K) \neq 0$ and $g$ is simple. Then two permissible metriss $C_{1}$ and $C_{2}$ induce the same affine connection if and only if there exists a scalar $\lambda$ such that $\lambda C_{1}=C_{2}$.

Proof. Let $T$ be defined as in Lemma 4.2. Now if $C_{1}$ is strongly equivalent to $C_{2}$ then by Lemma $4.2, T$ reduces to a scalar since Theorem 5.3 implies $\psi_{o}\left(C_{1}\right)$ is irreducible. The converse is trivial.

Q.E.D.

6.1. Invariant vector fields and the dominance of natural metrics. Aside from the case where $\%(G / K) \neq 0$ the condition that $a d_{\mp} K$ has inequivalent representations seems to be fairly restrictive. One would like a less restrictive condition in order to imply that $S$ leaves the spaces $n_{j}(C)$ and $n_{i}(B)$ invariant. In the following theorem we give a more likely condition which implies at least that $S$ leaves the spaces $h_{i}(B)$ invariant. Thus according to Theorem 4.3, $C<B$ for every permissible metric $C$ when this condition is satisfied. First we would like to point out that the example in $\$ 3.2$ illustrates tinat $B<C$ does not always hold. In fact in that example we had reducibility with respect to $C$ and irreducibility with respect to $B$. We now give an example where $C<B$ does not hold. Here we shall have reducibility with respect to $B$ and irreducibility with respect to $C$. We have seen (see $\$ 4.3$ ) that $C<B$ implies that $p_{0}(C)=p_{0}(B)$. The following example also illustrates that the strict inequality in the relation $\operatorname{dim} p_{0}(C) \leqq p_{0}(B)$ of Lemma 4.3 may hold. That is, in our example $0=\operatorname{dim} n_{\theta}(C)<\operatorname{dim} n_{0}(B)=1$.

Let $G=U(2)$, the $2 \times 2$ unitary group. Then the decomposition of $g$ as the direct sum of its center and its simple ideals becomes $g=g_{0}+g_{1}$ where $g_{0}$ is one-dimensional with basal element $X_{0}$ and $a_{1}$ is 3 -dimensional with basal elements $X_{1}, X_{2}, X_{3}$. The basis of $\mathrm{g}_{1}$ may be chosen so that $\left[X_{1}, X_{2}\right]=X_{3}$, $\left[X_{2}, X_{3}\right]=X_{1},\left[X_{2}, X_{1}\right]=X_{2}$.

Now let $K=e$ so that $b=$. Now it is clear that we may choose $B$ so that $X_{i}, i=0,1,2,3$ forms an orthonormal set. It is obvious also that $\Omega_{0}=\eta_{0}(B)$ 
and $g_{1}=\mathfrak{p}_{1}(B)$.

Now since $K=e$, any metric $C$ is permissible and hence every positive definite (with respect to $B$ ) operator $S$ may be obtained.

Now given $C$ then by Lemma 4.3 either $\operatorname{dim} p_{0}(C)=1$ or 0 . We assert that in case $\operatorname{dim} p_{0}(C)=0, G$ is irreducible. Indeed if $G$ were reducible then dimension-wise the only possible decomposition is $\mathfrak{p}=\mathfrak{p}_{1}(C)+\mathfrak{p}_{2}(C)$ where $\operatorname{dim} \mathfrak{p}_{1}(C)^{\prime}=\operatorname{dim} \mathfrak{p}_{2}(C)=2$. On the other hand by Lemma $3.4 \mathrm{~A}, \mathfrak{p}_{1}(C)$ and $\mathfrak{p}_{2}(C)$ are subalgebras of $g$. But since $G$ is compact $\mathfrak{p}_{1}(C)$ and $\mathfrak{p}_{2}(C)$ must be commutative. Since $g$ contains a non-zero center this easily implies the existence of a three dimensional commutative subalgebra of $\mathrm{g}$. This, however, is a contradiction.

It suffices then to exhibit a metric $C$ for which $\mathfrak{p}_{0}(C)=0$. Now if $q(C)$ and $q(B)$ are defined as in $\S 4.3$ then as we have seen there $q(B) \subseteq q(C)$. Since $q(B)=g_{1}$, to show $p_{0}(C)=0$ it suffices to choose $C$ so that $q(C)$ contains a vector not in $g_{1}$. Let $S$ be an operator on $g$ defined as follows: $S X_{0}=X_{0}+X_{3}, S X_{1}=2 X_{1}$, $S X_{2}=X_{2}, S X_{3}=2 X_{3}+X_{0}$. It is easy to verify that $S$ is positive definite with respect to $B$ and hence defines $C$. We observe that $S^{-1} X_{3}=X_{3}-X_{0}$. Now let $Y=a_{X_{1}} X_{2} \in q(C)$. Then $Y$

$$
\begin{aligned}
& =\left(S^{-1} D_{X_{1}} S+D_{X_{1}}-S^{-1} D_{S X_{1}}\right) X_{2} \\
& =X_{0} .
\end{aligned}
$$

Since $X_{0}$ is not in $g_{1}$ we conclude that $g$ is irreducible.

Now the condition that we shall impose on $\mathfrak{g}$, roughly speaking, produces the opposite situation from the one above. The condition is that the space of vectors in $\mathfrak{p}$ which are point-wise fixed by $a d_{\mathfrak{p}} K^{*}$ should be at most one dimensional." We shall show that this always implies $C<B$ for all $C$ when $p$ is chosen properly. In order to prove this we have to appeal to the results in [3]. However, these results were obtained under the additional hypothesis that $g$ acts effectively on $G / K$. Hence we shall have to make this assumption. However, we wish to point out that the results of [3], especially Theorem 4, can be modified so as to yield the following theorem without the assumption of effectiveness.

Now we choose $\mathfrak{p}$ so that the ideal $\mathfrak{g}(\mathfrak{p})=\mathfrak{p}+[\mathfrak{p}, \mathfrak{p}]$ in $\mathfrak{g}$ is equal to $\mathfrak{g}$. In

7) $K^{*}$ is the identity component of $K$. 
[3] we called $\mathfrak{p}$ pervasive in $\mathfrak{g}$ if $\dot{g}(\mathfrak{p})=\mathfrak{g}$. When $\mathfrak{g}$ acts effectively on $G / K$ we showed (Theorem 4 and Corollary 5 ) that a necessary and sufficient condition that $p$ be pervasive in $g$ is that $B$ can be extended to an invariant (under $a d G$ ) bilinear form $B^{*}$ on $\mathfrak{g}$ such that $\mathfrak{p}$ is orthogonal to ${ }^{*}$. In such a case $B^{*}$ is necessarily unique and non-singular on $\mathfrak{g}$. As mentioned in [3] even though the unique $B^{*}$ is non-singular and of course positive definite on $p$ it is not necessarily true that $B^{*}$ is positive definite on $g$. We now have

Theorem 6.1. Assume 8 acts effectively on $G / K$. Let $B^{*}$ be a non-singular invariant (under adG) bilinear form on $\mathrm{g}$ which is non-singular on ${ }^{*}$ and which is positive definite on the ortho-complement $\mathfrak{p}$ to $*$. Let $B$ be the restriction of $B^{*}$ to p.

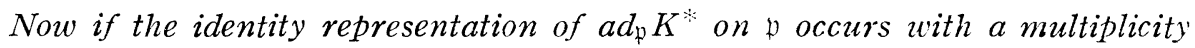
of at most one then $C<B$ for any permissible metric $C$.

Proof. Now let $p_{1} \cong \mathfrak{p}$ be a subspace invariant under $\psi_{o}(B)$. We must show then that $p_{1}$ is invariant under $\psi_{o}(C)$ where $C$ is any permissible metric. By Theorems 2.3 and 3.3 it suffices then to show that $\mathfrak{p}_{1}$ is invariant under $S$.

Now let $p_{2}$ be the orthocomplement to $\mathfrak{p}_{1}$ relative to $B$. We may now apply the results of [3] (in particular Theorem 5). Let $g_{i}=p_{i}+\left[p_{i}, p_{l}\right]$ where $i=1,2$. Then $\mathfrak{g}_{1}, g_{2}$ are ideals in $\mathfrak{g}$ where $\mathfrak{g}=\mathfrak{g}_{1} \oplus \mathfrak{g}_{2}$ and $\mathfrak{f}_{1}, \mathfrak{f}_{2}$ are ideals in $\mathfrak{f}$ where $\mathfrak{f}=\mathfrak{f}_{\mathfrak{t}}$ (千) $\mathfrak{f}_{2}$ and $\mathfrak{f}_{1}=g_{1} \cap$ and $\mathfrak{t}_{2}=g_{2} \cap *$ Let $K_{1}$ and $K_{2}$ be groups corresponding to $\mathfrak{f}_{1}$ and $\mathfrak{f}_{2}$. Now by our hypothesis either $K_{1}$ or $K_{2}$ must have the property that $a d_{\mathfrak{p}} K_{i}$ leaves no non-zero vector fixed in $\mathfrak{p}_{i}$. Since otherwise 2 linearly independent vectors would be invariant under $a d_{p} K^{*}$. Assume without loss that $i=1$. Thus the subspace $\mathfrak{p}_{2}$ is characterized as the set of fixed vectors for $a \vec{a}_{p} K_{1}$. Since $S$ commutes with $a d_{p} K^{*}$ it follows that $p_{2}$ and hence $p_{1}$ is left invariant by $S$.

Now it is obvious that if the identity representation of $a d_{p} K^{*}$ occurs at all on $p$ then there exists a non-singular invariant vector field on $G / K^{*}$. Hence we have

Corollary 6.1. Let $B$ arise from $a B^{*}$ in the manner of Theorem 6.1. Then if there exists no non-singular invariant vector field on $G / K$ and $K$ is connected, $C<B$ for all permissible metrics $C$. 


\section{BiBLIOGRAPHY}

[1] B. Kostant, Holonomy and the Lie algebra of infinitesimal motions of a Riemannian manifold, Trans. Amer. Math. Soc., vol. 80 (1955), pp. 528-542.

[2] B. Kostant, On differential geometry and homogeneous spaces, I. Proc. Nat. Acad. Sci., vol. 42 (1956), pp. 258-261.

[3] B. Kostant, On differential geometry and homogeneous spaces, II. Proc. Nat. Acad. Sci.. vol. 42 (1956), pp. 354-357.

[4] K. Nomizu, Invariant affine connections on homogeneous spaces, Amer. Jour. Math., vol. 76 (1954), pp. 33-65.

[5] A. Lichnerowicz, Espaces homogènes riemannien et reductibilité, Comptes Rendus, vol. 242 (1956), pp. 1410-1413.

[6] A. Lichnerowicz, Sur la réductibilité des espaces homogènes riemanniens. Comptes Rendus, vol. 243 (1956), pp. 640-642.

[7] Y. Matsushima and J. Hano, Some studies on Kaehlerian homogeneous spaces, Nagoya Math. J., vol. 11 (1957), pp. 77-92.

\section{Berkeley, California}

Added in proof.

[8] A. Lichnerowicz, Transformations affine et holonomie, Comptes Rendus, Vol. 244 (1957), pp. $1868-1870$. 